\title{
Transactive Control of Air-Conditioning Systems in Buildings for participation in Singapore's Demand Response Market
}

This paper was downloaded from TechRxiv (https://www.techrxiv.org).

\section{LICENSE}

CC BY-SA 4.0

SUBMISSION DATE / POSTED DATE

$16-01-2022$ / 20-01-2022

\section{CITATION}

Chandra, Rohit; Radhakrishnan, Krishnanand Kaippilly; Panda, Sanjib Kumar (2022): Transactive Control of Air-Conditioning Systems in Buildings for participation in Singapore's Demand Response Market. TechRxiv. Preprint. https://doi.org/10.36227/techrxiv.18515000.v1

DOI 


\title{
Transactive Control of Air-Conditioning Systems in Buildings for participation in Singapore's Demand Response Market
}

\author{
Rohit Chandra, Student Member, IEEE, Krishnanand Kaippilly Radhakrishnan, Member, IEEE and Sanjib Kumar \\ Panda, Fellow, IEEE
}

\begin{abstract}
Air-conditioning and mechanical ventilation (ACMV) systems account for major electrical demand within buildings. These systems have large thermal time constant and can be utilized as thermal energy storage. This paper is focused on participation of commercial buildings in Singapore's Demand Response (DR) programme by adjusting electricity demand of ACMV systems for financial incentive and power grid benefits. A novel Transactive Energy-based virtual market is proposed for a building where multiple zone agents transact to purchase conditioned-air from central ACMV system. These zone agents consider forecasts of occupancy and outdoor temperatures to submit bids for conditioned-air corresponding to increasing electricity market prices, using a novel dynamic programming (DP) approach to utilize the temporal flexibility in cooling demand through pre-cooling. ACMV electricity demand bid is calculated based on net conditioned-air bids by using existing accurate building models in whole-building energy modeling (BEM) platforms corresponding to increasing electricity prices for submission to Singapore's DR market. Case studies are performed in MATLAB-EnergyPlus co-simulation environment on a benchmark five zone building model. Results show that the proposed control is effective in providing DR according to real Singapore market price signals while respecting occupants' thermal comfort preferences and incentive from DR market participation can be lucrative.
\end{abstract}

Index Terms-Transactive control, demand response, central air conditioning, buildings.

\section{INTRODUCTION}

In the United States of America, commercial buildings account for around $35.7 \%$ of the total electricity consumption in 2019 [1]. Commercial sector consumes around 37.3\% of total electricity in Singapore in 2019 [2]. Bulk of electricity consumption (i.e. 70\%) in commercial buildings in Singapore was reported to occur in centralized air conditioning and mechanical ventilation (ACMV) systems in 2013 [3]. ACMV systems in buildings condition large volume of indoor air. Changes in cooling effort from ACMV system do not affect the temperatures instantly because of the associated large thermal time constant. This means that the buildings can act like a thermal energy storage device and provide flexibility

This research is supported by the National Research Foundation, Prime Minister's Office, Singapore under its Campus for Research Excellence and Technological Enterprise (CREATE) programme.

Rohit Chandra and Sanjib Kumar Panda are with the Department of Electrical and Computer Engineering, National University of Singapore, 119077, Singapore (e-mail: rohitchandra@u.nus.edu; eleskp@nus.edu.sg)

Krishnanand Kaippilly Radhakrishnan is with the Berkeley Education Alliance for Research in Singapore, CREATE Tower, 1 Create Way, \#11-00, 138602, Singapore (e-mail: krishnanandkr@u.nus.edu) in electrical demand [4]. Data centres also spend significant quantity of electricity on air conditioning [5].

This demand flexibility can be utilized to provide demand response (DR) services to the electrical utility. Energy Market Authority (EMA) of Singapore has established a Demand Response programme that enables consumers to reduce their electricity demand voluntarily, in exchange for a share in the system-wide benefits, in terms of reduction in wholesale energy prices as a result of their actions [6]. Usually, industrial consumers with chemical storage tanks are utilized for DR participation [7]. Transactive Energy (TE), defined as "a set of economic and control mechanisms that allows the dynamic balance of supply and demand across the entire electrical infrastructure using value as a key operational parameter", has been proposed as an overarching approach for DR control [8]. The merits of such an approach include full use of DR potential, certain system response, efficiency and privacy protection [9]. The demand side bidding concept in EMA's DR programme aligns well with the TE principles [10].

Centralized ACMV systems have been identified as major resources for managing the electricity demand curves because of their concentrated and high electrical demand. Yoon et al. presented a dynamic DR controller (DDRC) based on customer specified threshold for adjusting the temperature setpoints of heating ventilation and air-conditioning (HVAC) system corresponding to electricity retail prices [11]. This method is easy to implement but only considers pre-determined thresholds, thus making the approach static in nature. The drawback is that in scenarios with consistent high electricity prices, the temperature set-points may keep on increasing to high values, leading to excessive discomfort. Detailed characteristics of the HVAC components (such as interdependencies among subcomponents, effect of humidity, etc.) are not considered, which makes the demand response non-linear [12] and may also reduce HVAC operational efficiency. Reynolds et al. presented a multiple hidden layered artificial neural networks (ANN) for data-driven model of HVAC controlled by Genetic Algorithm (GA) [13]. ANN-based modeling for HVAC system reduces the explainability of the HVAC model and heuristics based control may not guarantee a control effort close to optimal value. Young-Jin Kim in [14] proposed a generalized ANN to develop piece-wise linear models for variation of indoor temperatures with changes in power input to HVAC systems. A large quantity of operational data is required for training ANN which can be avoided by use of existing BEM models 
such as EnergyPlus [15].

An HVAC fan-chiller model is presented by Daher et al. [17] where variation in chiller power consumption due to direct HVAC supply fan speed control is utilized for DR. With interdependent operation among the HVAC components, the reduction in fan power may cause change in chiller operating points and additional losses. Economic model predictive control (EMPC) for price-responsive optimal scheduling of HVAC systems is presented by Ostadijafari et al. in [18]. The modeling in this article is limited to linearized HVAC sub-component models to avoid the complexities associated with non-linear models, which reduces the accuracy of control. Hao et al. in [19] presented a hierarchical TE market based approach for demand management from commercial buildings through HVAC control. This paper considers detailed linearized models for HVAC components. Further, the dynamic effect of cooling effort on subsequent operation is not considered. Thus, there is a need to develop multi-step market mechanism for DR from ACMV systems which can be integrated and tested with detailed and accurate platforms that can represent the dynamics of cooling effort for the whole building.

Considering the challenges in running experiments in live building environment, integrated design of buildings with whole-building energy modeling (BEM) software has gained popularity [20]. Analysis of 7,100 projects submitted from 2013 to 2015 shows that $61 \%$ of projects use BEM [20]. EnergyPlus is US DOE's open-source BEM engine which embodies the state-of-the-art in BEM knowledge and is used by $10 \%$ of projects. It implements detailed building physics for air, moisture, and heat transfer. EnergyPlus analysis results are also accepted for compliance with energy standards and utility incentives [20]. Other BEM software include TRACE 700, TRNSYS, DesignBuilder, and Modelica.

In this paper, a novel TE-based control scheme is proposed for ACMV systems, considering occupants' thermal comfort preferences. This scalable control method utilizes decentralized computation through multiple zone variable air volume (VAV) control agents representing the zone occupant's thermal comfort flexibility through bidding in a virtual market to purchase conditioned air (i.e. cooling effort). The VAV agents change their cooling demand patterns in response to multiple time-step forecasts for peaks in electricity prices. The ACMV system is modeled as machine that consumes electrical energy to produce conditioned air to be sold to zones. Net ACMV electricity demand bid is calculated based on net conditioned air bids from all zones by using existing accurate building models in BEM platforms corresponding to increasing electricity prices, for submission to EMA's DR market. Further, the general requirements of Singapore EMA's DR program are followed.

Compared to related works, the challenges in modeling for ACMV system and occupants' comfort are addressed through a novel hybrid modeling approach in this paper. Zone thermal dynamics have linear models to support dynamic programming based bidding strategy. Linear models may lead to slight deviations in zone temperatures $\left( \pm 1^{\circ} \mathrm{C}\right)$ which are acceptable for occupants' comfort. ACMV system components have detailed non-linear models from EnergyPlus for accurate estimation of electricity demand to ensure conformation to DR bids. This is an extension on our earlier work [21], with addition of novel dynamic programming (DP) based bidding approach for VAV agents and integration of EnergyPlus platform. The proposed control scheme is also flexible to be integrated with other BEMs platforms as needed. Groups of buildings being serviced by a common district cooling system (DCS) may also implement proposed control scheme.

The major contributions of this paper are:

(a) a TE-based virtual market is proposed where individual zone agents transact to purchase conditioned air (i.e. cooling effort) to respond to electricity market prices for EMA DR market participation,

(b) dynamic programming based bidding strategies (along with pre-calculation approach for faster solution) are presented for zone agents which enables pre-cooling for higher DR capacity (i.e. 56.67\% DR for 1 hour);

(c) detailed EnergyPlus models are integrated for electricity demand estimation which lead to accurate control and operation, and

(d) case studies are reported based on Singapore electricity market prices and the proposed approach is shown to perform well with real prices. Further, DR revenue is also evaluated.

The rest of the paper is organized as follows, Section II discusses Singapore EMA's DR programme, Section III explains the modeling for ACMV system components and Section IV explains the proposed virtual TE market based control. Subsequently, Section V discusses co-simulation studies and test results which validate the efficacy of the proposed approach. Finally, Section VI concludes this paper and proposes scope for future work.

\section{SingAPORE EMA's DEMAND RESPONSE}

This programme enables contestable consumers (i.e. consumers with $>2000 \mathrm{kWh}$ electricity consumption per month) able to offer a load curtailment of at least $0.1 \mathrm{MW}$ (including aggregated loads) to participate in the wholesale electricity market through demand-side bidding [22]. The consumers indicate their "willingness to consume" at various electricity price points for upcoming market period by adjusting their loads in response to real-time supply and demand conditions [10]. The forecast of wholesale electricity prices for 72 market periods (30 minutes each) available on Singapore Energy Market Company's website [23] can be utilized to plan these demand bids.

The submitted demand bids for a particular market trading period would include : (i) total base load (in MW) for the time period, (ii) a sequence of energy curtailment and price quantity tranches (i.e. demand bid), and (iii) the linear ramp rates $(\mathrm{MW} / \mathrm{min})$ as determined for the participant.

EMA DR Incentive: One-third share of the additional consumer surplus (ACS) generated as a result of load curtailments dispatched is paid to the participants. The incentive payment will be allocated to the eligible participants based on their respective energy curtailment amount (load curtailment quantity $\left.\left(\mathrm{LCQ}_{h}^{i}\right)\right)$ in accordance with their demand dispatch schedule. The incentive payment per unit of load curtailment (MWh) is 
TABLE I

RECENT EMA DR MARKET ACTIVATION INSTANCES [23]

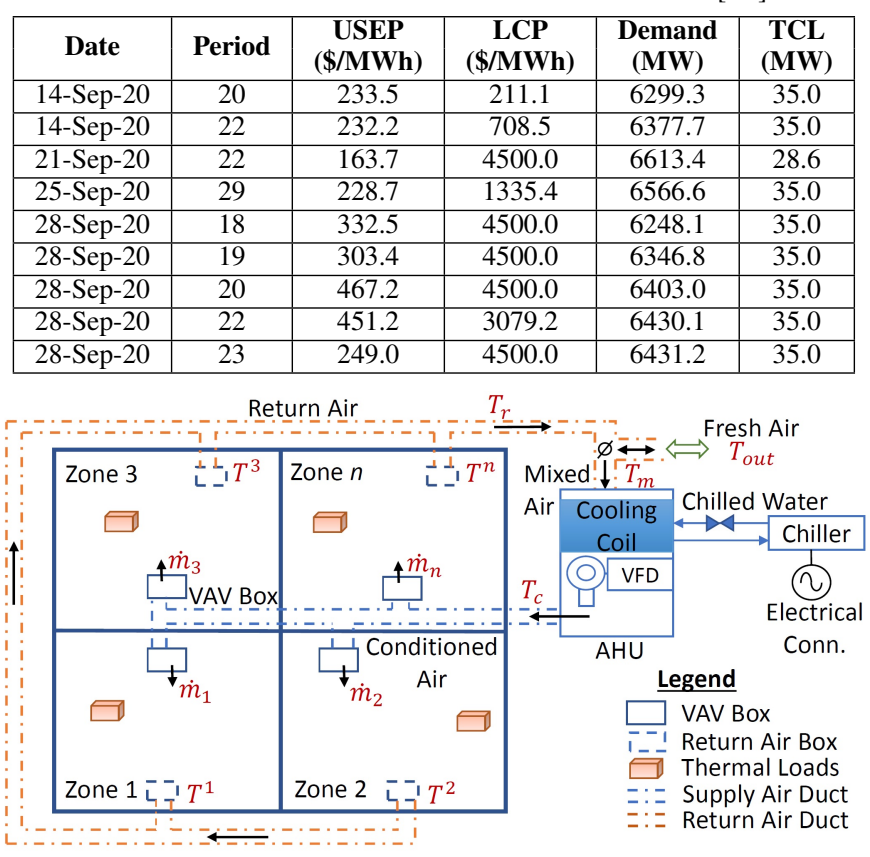

Fig. 1. Typical ACMV system schematic

calculated by the market clearing engine and referred as load curtailment price (LCP), capped at $\$ 4500$ (Singapore Dollars). The load curtailment settlement credit (LCSC) to $i^{\text {th }}$ compliant participant is calculated using LCP as per (1).

$$
L C S C_{h}^{i}=L C P_{h} \times L C Q_{h}^{i}
$$

The recent instances of activation of demand curtailment for EMA DR market are summarized in Table I based on data available from Energy Market Company [23]. It can be observed from Table I that majority of DR activations occur during market periods numbered $18-23$ (i.e. 09:00-11:30), which are the peak demand periods for Singapore. Further, it is observed that the cleared load curtailment prices (LCP) are much higher in comparison to the energy prices (USEP), thus making the prospect of DR market participation an attractive opportunity.

\section{Methodology}

A typical ACMV system schematic is shown in Figure 1. The ACMV components are VAV boxes, air-handling unit (AHU) and chiller with variable frequency drives (VFD).

\section{A. Extended Thermal Model for ACMV Zones}

A typical ACMV system with $n$ zones is considered. For each indoor zone, $i=1,2,3 \ldots n$, there is a VAV controller associated with it for temperature control. The linear thermal model for the ACMV zones can be generalized in the form of (2) as per [19].

$$
T^{i}(t+1)=b_{1}^{i} T^{i}(t)+b_{2}^{i} T_{o}(t)+b_{3}^{i} q^{i}(t+1)+b_{4}^{i} q^{i}(t)+b_{5}^{i}
$$

Here, $b_{1}^{i}-b_{5}^{i}$ are constants, $T_{o}(t)$ is the outdoor temperature, and $q^{i}(t)=-q_{c}^{i}(t)$ i.e. the cooling effort, which can be expressed based on Newton's Law of Cooling as:

$$
q_{c}^{i}(t)=c_{a} \dot{m}^{i}(t)\left[T^{i}(t)-T_{c}(t)\right]
$$

TABLE II

IDENTIFIED PARAMETERS FOR 5 ZONES IN THE ENERGYPLUS MODEL

\begin{tabular}{|c|c|c|c|c|c|c|}
\hline \multirow{2}{*}{$\begin{array}{c}\text { Zone } \\
(i)\end{array}$} & \multicolumn{7}{|c|}{ Parameters } \\
\cline { 2 - 7 } & $a_{1}^{i}$ & $a_{2}^{i}$ & $a_{3}^{i}$ & $a_{4}^{i}$ & $a_{5}^{i}$ & $a_{6}^{i}$ \\
\hline 1 & 0.4661 & 0.079 & -0.00087 & 2.779 & 1.1042 & 9.2565 \\
\hline 2 & 0.4687 & 0.0603 & -0.0015 & 3.0178 & 0.9923 & 9.366 \\
\hline 3 & 0.3805 & 0.1019 & -0.00111 & 4.3466 & 1.2941 & 10.5247 \\
\hline 4 & 0.4687 & 0.0603 & -0.0015 & 3.0178 & 0.9923 & 9.366 \\
\hline 5 & 0.4551 & 0.0304 & -0.00079 & 2.4662 & 1.4043 & 10.7471 \\
\hline
\end{tabular}

where, $c_{a}$ is the specific heat capacity of air, $\dot{m}^{i}(t)$ is the conditioned air flow rate, and $T_{c}(t)$ is the conditioned air temperature. The linear model in (2) can be utilized for regression analysis on experimental data to find the parameters $\left(b_{1}^{i}-b_{5}^{i}\right)$ for a given zone. This linear model may then be used to predict the thermal response $\left(T^{i}(t+1)\right)$ for the next time step for a given set of inputs i.e $T_{o}(t), q^{i}(t+1)$, and $q^{i}(t)$. On extrapolating this model to predict zone temperatures $\left(T^{i}(t+1), T^{i}(t+2), \ldots T^{i}(t+n)\right)$ for multi-steps, the existing model fails. This is especially the case when the building is not uniformly occupied at certain times of the day [18].

Hence, an extended linear thermal model for the ACMV zones is proposed to include effect of occupants $\left(O^{i}(t)\right)$ and corresponding electric equipment $\left(E^{i}(t)\right)$ schedules. From regression analysis, it is also observed that only one cooling effort term $\left(q^{i}(t)\right)$ is significant and the heat transfer across zones is also insignificant. The extended model is shown in (4). Other terms in this equation are zone indoor temperature $\left(T^{i}(t)\right)$, outdoor temperature $\left(T_{o}(t)\right)$, cooling effort $\left(q^{i}(t)\right)$ for the time-step $t$ and predicted zone indoor temperature $\left(T^{i}(t+1)\right)$ for next time-step $t+1$.

$$
\begin{aligned}
T^{i}(t+1)=a_{1}^{i} T^{i}(t)+a_{2}^{i} T_{o}(t)+a_{3}^{i} q^{i}(t) \\
+a_{4}^{i} E^{i}(t)+a_{5}^{i} O^{i}(t)+a_{6}^{i}
\end{aligned}
$$

\section{B. Zone Thermal Parameter Identification}

The "5ZoneAirCooled.idf" model from EnergyPlus [15] based on "Small Office Building" benchmark [24] is utilized for simulation studies. This model has single story building with floor space of $463.6 \mathrm{~m}^{2}\left(5000 \mathrm{ft}^{2}\right)$ divided into 5 zones with individual VAV agents and has a central air handling system with single duct.

Customization for Singapore buildings: This model is modified to represent Singapore (or tropical) climatic conditions where heating coils are not present. Further, EnergyPlus Singapore weather data [25] is used for analysis.

To enable direct control of conditioned air $\left(\dot{m}^{i}(t)\right)$ and corresponding cooling effort $\left(q^{i}(t)\right)$ for each zone $(i)$, a ConstantVolume:NoReheat air terminal [26] is used. For the extended thermal model (4), cooling effort $\left(q^{i}(t)\right)$ is varied independently from the indoor temperatures $\left(T^{i}(t)\right)$ using a EnergyPlus Energy Management System (EMS) program. The effect of each term on the right hand side on updated temperature $\left(T^{i}(t+1)\right)$ is studied. Output data from modified EnergyPlus case is regressed in MATLAB using Statistics and Machine Learning Toolbox to find coefficients for (4) at 1 hour time-steps. Identified model coefficients for the five zones are shown in Table II. It was observed that for all coefficients, $p$-value $\leq 0.05$ and $R$ - squared $\geq 0.95$ for all the five fitted models. Fig. 2 depicts the comparison 


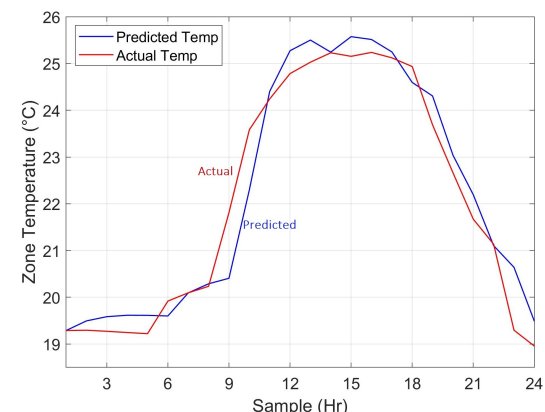

Fig. 2. Comparison of predicted and actual indoor temperatures

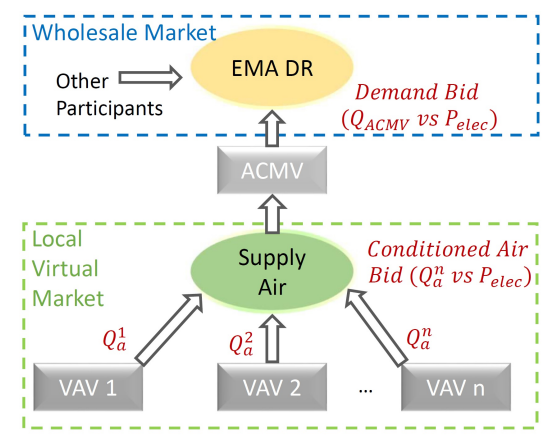

Fig. 3. Proposed virtual Transactive Market based control schematic

between the EnergyPlus output temperature and the predicted temperature using identified model parameters for 24 outof-sample data points for zone 1 . It can be observed that predictions match well and the error is less than $1{ }^{\circ} \mathrm{C}$ (i.e. $<4 \%$ ) for predictions up to 24 steps in future, such small variations are not expected to significantly affect the occupant thermal comfort. Thus, this extended model provides suitable accuracy while maintaining linear characteristics which are important to develop the bidding strategy for zone VAV agents to represent the flexibility in cooling demand in zones based on occupant thermal comfort.

\section{ACMV System Model}

Control focused models for air handling unit (AHU) fans and chillers have been proposed in literature [19]. However, these models are linear approximations and do not consider effects of humidity on temperature and other parameters. For this work, the EnergyPlus engine, which considers detailed models for ACMV system sub-components and is able to model such interdependencies, is directly used. An example of non-linear models in EnergyPlus for absorption chiller is as following. The percentage of electric input power is a quadratic function of the part-load ratio $(P L R)$ [15]. The $P L R$ is simply the actual cooling effect produced by the chiller divided by the maximum cooling effect available.

$$
\text { ElectricInputRatio }=C_{1}+C_{2} P L R+C_{3} P L R^{2}
$$

where, $C_{1}, C_{2}$, and $C_{3}$ are constant coefficients.

\section{TRANSACTIVE MARKET BASED CONTROL}

A virtual TE market structure is proposed for transactions on conditioned air, as shown in Figure 3. Here, the zone VAV agents compete to buy conditioned air $\left(Q_{a}^{i}\right)$ according to occupant preferences. The market clearing steps are:

1) VAV agents calculate and submit their optimal demand curve for conditioned air $\left(Q_{a}^{i}\right)$ corresponding to a range of current electricity prices $\left(P_{\text {elec }}\right)$ and forecast of electricity prices (i.e. multi-step control).

2) Total conditioned air demand for ACMV system corresponding any price is the sum of all VAV supply air bids $\left(Q_{a}=\sum_{i=1} Q_{a}^{i}\right)$.

3) The AHU fan and chiller electricity demand is calculated by the EnergyPlus engine based on net supply air bids $\left(Q_{a}\right)$.

4) The net electricity demand for ACMV system $\left(Q_{A C M V}\right)$ plotted corresponding to increasing electricity prices $\left(P_{\text {elec }}\right)$ is the net electricity demand curve for ACMV system.

5) Based on the current electricity prices, the supply air quantities are cleared for individual zone agents from submitted bids, and ACMV electricity demand is picked from the aggregated demand curve.

\section{A. Multi-step Bidding Strategy for VAV agents}

The EMA's DR market clears one step at a time. Therefore, the virtual ACMV TE market also needs to be cleared step-bystep. The challenge for the VAV agent is to submit bids for one step market, while considering the operation for $n$ time-steps in future. In order to include the effect of present decision of cooling effort on future cooling effort requirements, discretetime Dynamic Programming (DP) approach [27] is proposed in this work. The DP approach aligns perfectly with the bidding requirements for the EMA's DR market and is suitably utilized. This approach provides a good trade-off between computational complexity (limited by discrete-time formulation and pre-calculation) and guaranteed optimal control effort through DP. The DP equation [27] expresses the balance each optimal decision must meet between immediate and future cost as shown in (6).

$$
J_{t}\left(u_{t}\right)=\min _{u_{t}}\left[g\left(x_{t}, u_{t}\right)+J_{t+1}\left(f\left(x_{t}, u_{t}\right)\right)\right]
$$

Here, $J_{t}$ represents the optimal policy at step $t, g\left(x_{t}, u_{t}\right)$ represents the immediate cost, and $J_{t+1}\left(f\left(x_{t}, u_{t}\right)\right)$ is future cost considering optimal decisions. For the control of ACMV system, this equation can be modified as in (7):

$$
\min C_{o p}(t+1: t+n)=C_{o p}(t+1)+\alpha C_{o p}(t+2: t+n)
$$

where, $C_{o p}(t+1: t+n)$ is the total cost of operation from time step $t+1$ to $t+n$. Also, $C_{o p}(t+1)$ is the immediate cost of operation, and $C_{o p}(t+2: t+n)$ is the future cost of operation, and $\alpha$ is a constant added in (7) to facilitate the comparison in conflicting interests of immediate and future cost minimization. From the zone's thermal model, it is established that future operation costs $\left(C_{o p}(t+2: t+n)\right)$ are dependent on indoor temperature $(T(t+1))$. Thus, the future operation costs can be expressed as a function of indoor temperature $\left(\tilde{C}_{o p}(T(t+1))\right)$, given other parameters (such as outdoor temperatures and predicted electricity prices) remain same. The above equation (7) can be re-written as:

$$
\min C_{o p}(t+1: t+n)=C_{o p}(t+1)+\alpha \tilde{C}_{o p}(T(t+1))
$$

\section{1) Immediate Costs}

For any zone, the bid for supply air vs electricity prices is formed based on the proposed discomfort cost model. The 
cost of discomfort felt by the $i^{\text {th }}$ zone occupants (equivalent to comfort model in [28]) using occupant preference $\left(c_{i}\right)$ is represented as:

$$
C_{d i s}(t+1)=\frac{1}{2} c_{i}\left[T(t+1)-T_{\text {set }}\right]^{2}
$$

From the expression for indoor zone temperature $(T(t))$ in (2) for the $i^{t h}$ zone which can be simplified by dropping the index $i$ :

$$
\begin{aligned}
T(t+1)=a_{1} T(t)+a_{2} T_{o}( & t)+a_{3} q(t) \\
+ & +a_{4} E(t)+a_{5} O(t)+a_{6}
\end{aligned}
$$

The total cost of operation may be expressed as sum of cost of cooling effort and cost of discomfort felt by occupant.

$$
C_{\text {cool }}(t+1)=P_{c}(t) Q(t)
$$

Here, $P_{c}(t)$ is the market price for conditioned air (i.e. cooling effort $[Q(t)=q(t) \cdot \Delta t])$ for market clearing period $(\Delta t)$ from the ACMV system.

$$
\min _{Q(t)} C_{o p}(t+1)=C_{d i s}(t+1)+C_{\text {cool }}(t+1)
$$

Further, $Q_{\max }(t)$ and $Q_{\min }(t)$ are calculated based on the maximum $\left(T_{\max }\right)$ and minimum temperature $\left(T_{\min }\right)$ constraints set by the occupant. Equation (12) is the objective function for immediate operation cost individual VAV agents. The optimal value of cooling effort $(Q(t))$ leads to right trade-off between economy and comfort for given occupant preference $\left(c_{i}\right)$. It may be observed that this problem is a quadratic in $(Q(t))$.

\section{2) Future Costs}

The problem formulation for future costs considering $n-1$ steps in future (i.e. $t+2, t+3, \ldots t+n$ ) is as follows. The objective function is the sum of costs of cooling effort and discomfort for $n-1$ steps for a given starting indoor temperature $(T(t+1))$ as shown in (13):

$$
\min _{\bar{Q}_{c}} C_{o p}(t+2: t+n)=\sum_{h=t+2}^{t+n}\left[C_{d i s}(h)+C_{\text {cool }}(h)\right]
$$

with the optimization variable as optimal cooling effort $\left(\bar{Q}_{c}=\right.$ $\left.\left[Q_{t+2}, Q_{t+3}, \ldots Q_{t+n}\right]\right)$ for next $n-1$ steps, subject to indoor temperatures $(\bar{T}=[T(t+2), T(t+3), . . T(t+n)])$ over $n-1$ steps being maintained within maximum $\left(T_{\max }\right)$ and $\left(T_{\min }\right)$ limits set by the occupants (14).

$$
T_{\min } \leq T(h) \leq T_{\max } \forall h=t+2, t+3, \ldots t+n
$$

To estimate the future operation costs, it is assumed that the each step will have optimal operation. The task then involves calculating this set of optimal cooling effort values $\left(\bar{Q}_{c}\right)$.

\section{B. Solution for future operation costs for VAV agent}

A gradient descent based algorithm with modified method of handling linear constraints is proposed for calculation of optimal cooling effort policy $\left(\bar{Q}_{c}\right)$ for the future time-steps. The $2(n-1)$ inequality constraints (14) related to $T_{\max }$ and $T_{\min }$ are observed to be linear in $\bar{Q}_{c}$ using bootstrap (10) and can be interpreted as $n$ dimensional hyperplane. This

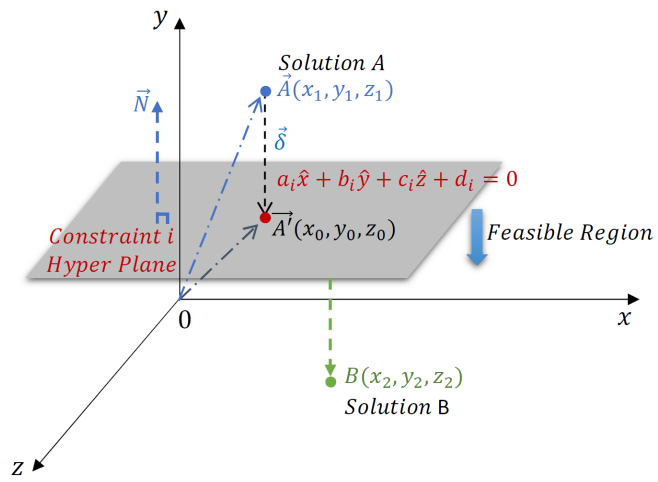

Fig. 4. Illustration of geometric interpretation of linear constraints

hyperplane represents a boundary of feasible region for the solution. A given solution $\bar{Q}_{c}$ can be interpreted as coordinates of a point $(A)$ in the $n$ dimensional space.

A simplified illustration is shown in Fig. 4. The plane corresponding to constraint $(i)$ is boundary of the feasible region for solution (below) and unsafe region (above). It can be observed that the solution represented as Point $\vec{A}$ falls outside the feasible boundary and solution represented as Point $\vec{B}$ falls within the feasible region. During the iterations of the gradient descent algorithm, the proposed solution $\vec{A}$ may be beyond any constraint hyperplane. We can bring this point back to the feasible region by taking the projection of $\vec{A}$ on the constraint hyperplane $(i)$ to get the point $\left(\vec{A}^{\prime}\right)$. Projection $\overrightarrow{A^{\prime}}$ is the intersection point of line parallel to normal vector $(\vec{N})$ for constraint hyperplane passing through the point $\vec{A}$ as shown in (15).

$$
\overrightarrow{A^{\prime}}=\vec{A}-(\vec{A} \cdot \vec{N}) \cdot \hat{N}
$$

The projected solution $\left(A^{\prime}\right)$ is optimal solution while respecting the constraint $i$. Therefore, a given gradient descent solution $(A)$ is projected on all violated constraint boundaries to ensure that gradient descent solution is feasible. Pre-calculation: Based on gradient descent, a mathematical model is developed for variation of future operation costs $\left(C_{o p}(t+2: t+n)\right)$ with change in current indoor temperature $(T(t+1))$. This model can be pre-calculated and utilized for solving (8) quickly.

\section{Calculation of VAV agent's Optimal Bid}

The overall optimization problem for time-step $(t)$ as in (8) is solved using a gradient descent approach to find optimal cooling effort $\left(Q_{c}^{i}(t)\right)$ (also expressed as conditioned air amount $\left(Q_{a}^{i}(t)\right)$ using (3)) subject to $T_{\max }$ and $T_{\min }$ constraints.

\section{Practical Implementability}

The proposed TE-based control may be implemented in non-invasive manner by integration with the existing building management system (BMS) in either of following two ways:

1) cleared quantities of conditioned air from virtual market for each zone may be directly used as set-point of conditioned air (i.e. flow rates) for zone VAV, or

2) calculated indoor zone temperatures corresponding to cleared conditioned air quantities may be used as the zone VAV temperature set-point. 


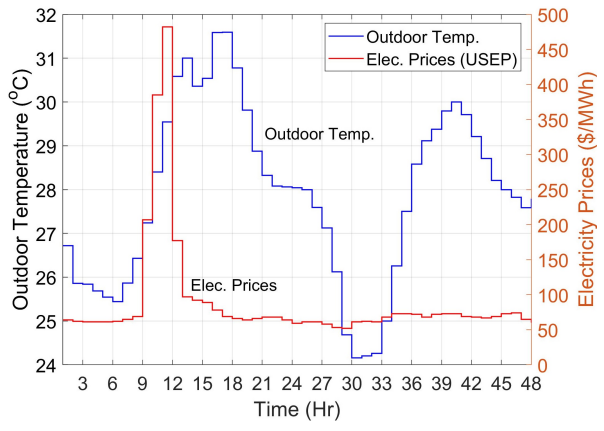

(a)

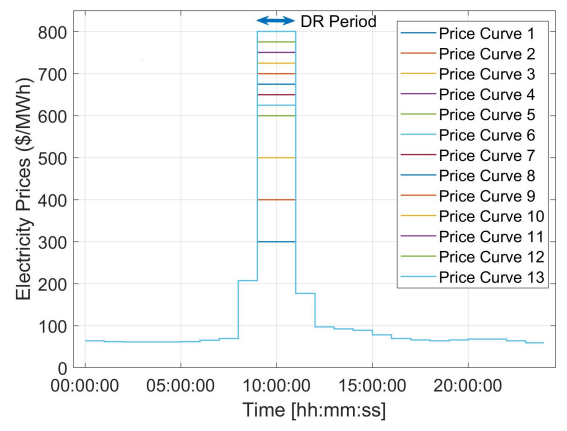

(b)

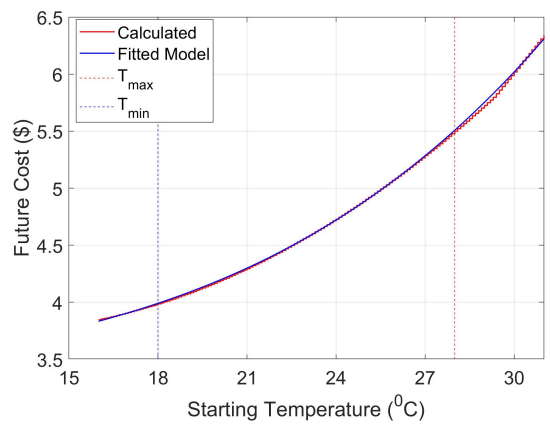

(c)

Fig. 5. Co-simulation parameters (a) Singapore electricity prices [23] for $28^{\text {th }}$ Sep. 2020 and outdoor temperature profile [25]. (b) Electricity price vectors with varying prices for 09:00-10:00 AM. (c) Effect of starting temperature on future costs for zone 1 bidding for 09:00-10:00 AM.

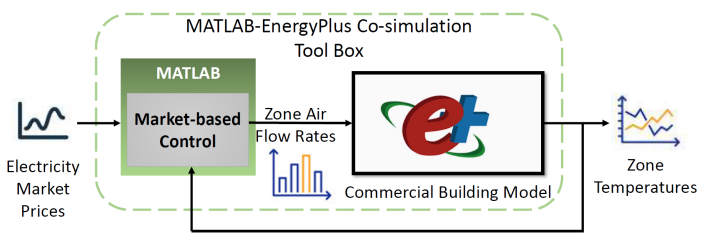

Fig. 6. MATLAB-EnergyPlus co-simulation set up

\section{Results AND Discussion}

Simulation studies are carried out in MATLAB-EnergyPlus co-simulation [29] environment which combines EnergyPlus with a MATLAB implementation of proposed TE market and agent bidding strategies, to test the proposed control scheme as shown in Figure 6.

\section{A. Co-simulation setup}

An ACMV system as shown in Figure 1 consisting of 5 zones, based on the modified 5zoneAirCooled model as discussed in III-B is considered. As the ACMV zones have large thermal time-constant and the wholesale electricity market also clears in half-hour periods, the reported zone parameters are used to develop a thermal model with time-period of 1 hour as shown in Table II.

"Small Office Building" model is simulated in EnergyPlus with a time-step of 3 minutes. The five zones have similar preferences for thermal comfort as $c_{i}=0.05$. The cleared quantities of conditioned air for each indoor zone from market agent in MATLAB are sent to the EnergyPlus simulation model at each simulation time-step and zone temperatures are collected as feedback as shown in Figure 6.

The maximum and minimum indoor zone temperature limits are set to 28 and $18^{\circ} \mathrm{C}$. These limits are set considering minor discomfort to occupants for an hour to provide DR. Occupancy $(O(t))$ and equipment $(E(t))$ schedules are taken from EnergyPlus. The electricity prices for Singapore (USEP) [23] for $28^{\text {th }}$ September 2020 and outdoor temperatures based on EnergyPlus Singapore weather data [25] considered in the studies are shown in Figure 5(a). The selected day had an activation of Demand Response (DR) and a large variation in electricity prices. The DR prices are shown in Table I.

\section{B. Experiments and results}

Three cases are simulated:

1) Baseline - Effect of electricity prices are not considered for ACMV control. Preference only to maintain comfort.

2) One step bidding - VAV agents bid on virtual market considering only the electricity prices for the next step (keep $\alpha=0$ ) and future costs are not considered [21].

3) Multi-step bidding - VAV agents bid considering 12 hours in the future with dynamic formulation (with $\alpha=2.5$ to maintain balance between immediate and future costs).

From the electricity prices (USEP) in Figure 5(a), it can be observed that peak electricity prices occur during the 09:0011:00 AM period. For calculation of the demand side bid from the ACMV system, various electricity price vectors as shown in Figure 5(b) are made by varying the peak prices for 09:0011:00 AM from 300-800 $\$ / M W h$. The electricity demand for bidding period 09:00-10:00 AM (market periods 19-20) can be calculated with reference to these price vectors. The variation of electricity demand from ACMV system for bidding period 09:00-10:00 AM corresponding to varying electricity prices would be electricity demand bid as discussed subsequently.

As discussed in Section IV-A, using the proposed thermal comfort model, agents representing VAVs can calculate the optimal value of cooling power $\left(Q_{c}\right)$ and supply air $\left(Q_{a}\right)$ corresponding to given rates of these quantities. In order to further simplify the operation, optimal quantity may be determined directly corresponding to electricity prices $\left(P_{\text {elec }}\right)$. The computational time for bidding and market clearing based on proposed control for a given time-step on Intel Core i5-8400 (3.8 GHz) computer is around 5 minutes, which is acceptable. The computational performance is also improved by precalculation of a model of future operation costs $\left(\tilde{C}_{o p}(T(t+1))\right)$ as discussed subsequently.

The variation of future operation costs $\left(\tilde{C}_{o p}(T(t+1))\right)$ with change in current indoor temperature $(T(t+1))$ for 9:00-10:00 AM period for zone 1 is shown in Figure 5(c). A $3^{r d}$ degree polynomial with constants $\left(\beta_{1}-\beta_{4}\right)$ as shown in (16) is used to approximate these models. It can be observed that the fitted model is able to represent the calculated trend.

$\tilde{C}_{o p}(T(t+1))=\beta_{1}[T(t+1)]^{3}+\beta_{2}[T(t+1)]^{2}+\beta_{3} T(t+1)+\beta_{4}$

The bids for conditioned air for all zones for 9:00-10:00 AM (i.e. peak price period) are shown in Figure 7(a). It may be observed that as the electricity prices increases, the VAV bids for conditioned air $\left(Q_{a}^{i}\right)$ reduce as expected. The variation of the cooling effort demand also follows the trend of conditioned air demand.

Based on the supply air $\left(Q_{a}^{i}\right)$ bids submitted by individual VAV zones, the net electricity demand bids for the ACMV 


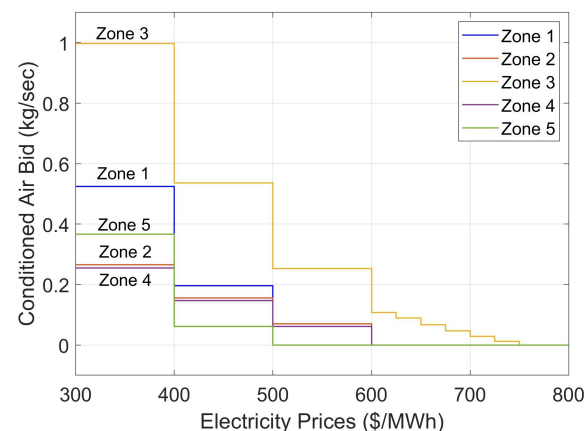

(a)

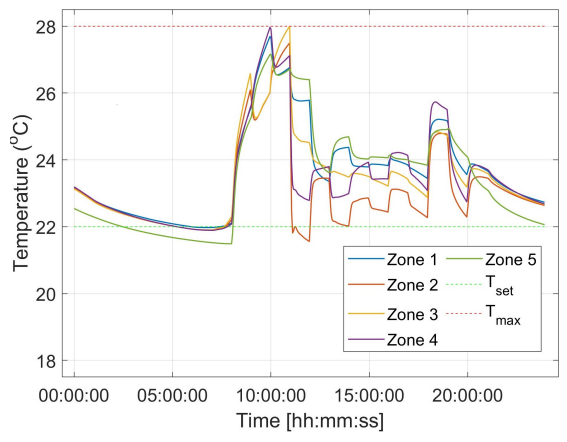

(d)

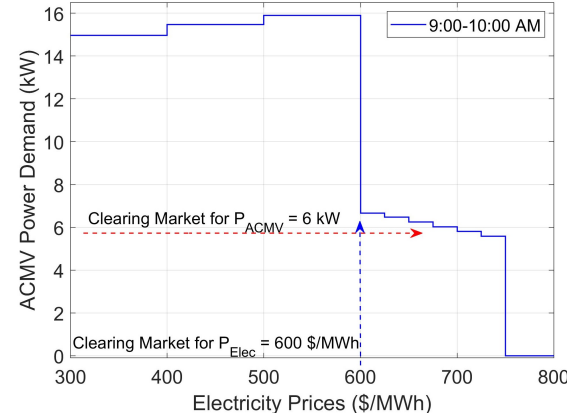

(b)

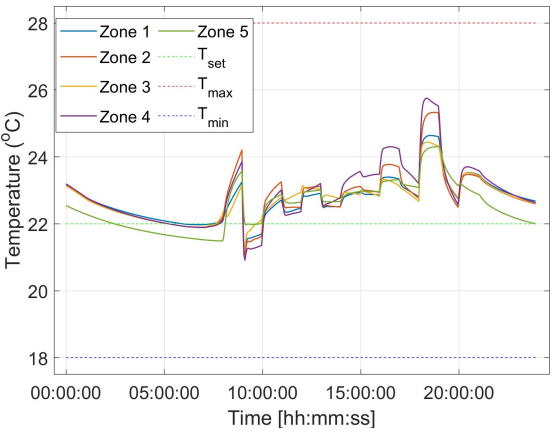

(e)

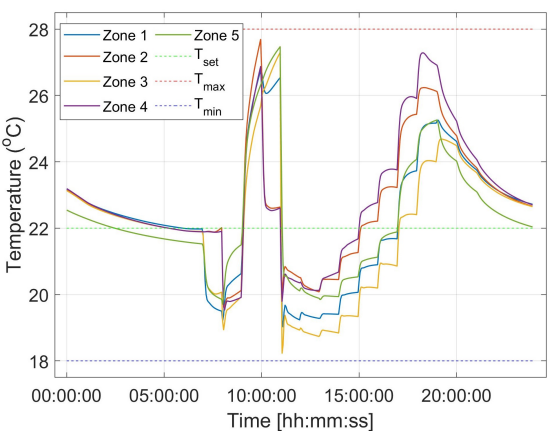

(c)

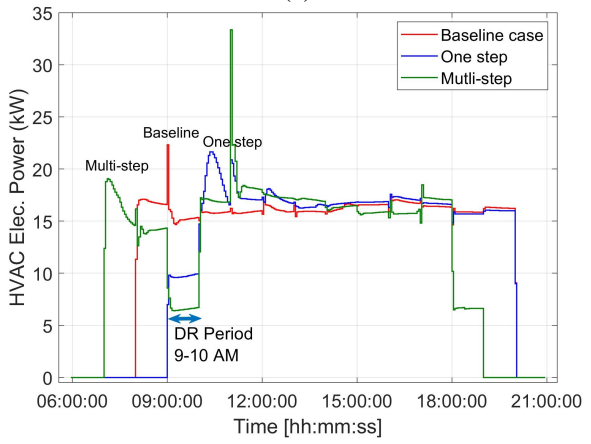

(f)

Fig. 7. Co-simulation results. (a) Comparison of conditioned air bids among the five zones. (b) Clearing of bids for DR or power demand reference following. (c) Variation of indoor temperature - Multi-step bidding. (d) Variation of indoor temperature - One step bidding. (e) Variation of indoor temperature for non-DR i.e. baseline case. (f) Variation in electricity consumption by the ACMV system.

system as shown in Figure 7(b) are calculated using the EnergyPlus engine. This curve may be directly used to represent the ACMV system demand through "willingness to consume" at various price points in the EMA DR market and other demand side management (DSM) markets.

It is observed from Figure 7(b) that the ACMV electricity demand remains close to $15 \mathrm{~kW}$ initially and then reduce significantly for the $600-750 \$ / \mathrm{MWh}$ price range. It is important to note that the reduction in electricity demand is not linear as the detailed non-linear EnergyPlus models for ACMV components are considered. This demand curve may also be utilized for following any demand reference signal as shown in Figure 7(b). Based on Figure 7(b), the operation of the ACMV is selected to follow the $600 \$ / \mathrm{MWh}$ bid, as there is significant reduction in electricity demand and the variation of indoor temperatures is within the set-points.

The variation of indoor temperatures for the different zones after clearing of the transactive market at each hourly timestep for 24 hours is shown in Figure 7(c). It may be observed that as the indoor temperatures are reduced during the 07:0009:00 AM period for pre-cooling and then there is increase during the target DR periods of 09:00-10:00 AM.

In order to isolate the effects of proposed dynamic programming based multi-step bidding strategy for VAV agents, a one-step bidding strategy case is simulated based on our earlier work [21]. The corresponding variation of indoor temperatures is shown in Figure 7(d). It can be observed that in absence of multi-step bidding, pre-cooling is not possible. To study the performance of the control under normal operating conditions, the corresponding indoor temperature profiles for non-DR activation i.e. baseline case are shown in Figure 7(e). It may be observed that under normal conditions, the proposed control operates the ACMV system to keep the zone temperature close to set-points. This result showcases the dynamic abilities of the proposed control to function in both normal and DR activation cases without the need for any operator intervention.

The variation in electricity consumed by the ACMV system over the day for three cases studied are compared in Figure 7(f). It may be observed that as desired there is drop in electricity consumption during 09:00-10:00 AM period. The electricity demand profiles in Figure 7(f) are analysed to calculate the electricity costs based on the electricity prices and the summary is presented in Table III. It is observed that the electricity costs are reduced on providing DR services as the electricity consumption is reduced from peak price periods and shifted to off-peak periods. The quantum of DR (i.e. LCQ) provided is also calculated for both one and multi-step bidding to be $5.5 \mathrm{kWh}(36.67 \%)$ and $8.5 \mathrm{kWh}(56.67 \%)$ in the target period 09:00-10:00 AM.

Benchmark for comparison: In the studies reported by Hao et al. in [19] for buildings in USA, the maximum reduction in HVAC demand in response to electricity market prices was $12.3 \%$ over few hours of operation for similar indoor temperature variation range $\left(18-28{ }^{\circ} \mathrm{C}\right)$. This study may be considered as comparable benchmark.

Commercial buildings usually have ACMV systems electrical demands up to few hundreds of kilowatts which can directly participate in DR market. Further, groups of buildings may also aggregate their LCQ to reach $0.1 \mathrm{MW}$ limit for EMA DR. Thus, the proposed multi-step market bidding approach is advantageous over our earlier proposed one-step bidding approach [21], at the cost of added computation. This is enabled by pre-cooling the zones from 07:00-09:00 $\mathrm{AM}$ as effect of the proposed dynamic programming based 
TABLE III

SUMMARY OF ELECTRICITY BILLS AND DR REVENUE

\begin{tabular}{|c|l|c|c|c|}
\hline Sr. No. & Case & $\begin{array}{c}\text { Electricity Bill } \\
24 \text { hours }(\$)\end{array}$ & $\begin{array}{c}\text { DR LCQ } \\
(\mathrm{kWh})\end{array}$ & $\begin{array}{c}\text { DR Revenue } \\
1 \text { hour }(\$)\end{array}$ \\
\hline 1 & Baseline & 29.99 & - & 0 \\
\hline 2 & One step & $26.4(-12 \%)$ & $5.5(-36.67 \%)$ & 24.75 \\
\hline 3 & Multi-step & $26.83(-10.5 \%)$ & $8.5(-56.67 \%)$ & 38.25 \\
\hline
\end{tabular}

approach which also leads to marginally higher thermal losses and marginally higher electricity consumption. For multi-step bidding case, a electrical demand peak lasting a few minutes is observed at 11:00 AM when the ACMV system is loaded again. This is expected as part of the in-rush demand for chillers.

Further, the cleared DR prices (LCP) for target DR period from Table I and DR quantum (LCQ) are utilized to calculate the DR revenue. It may also be observed from Table III that the DR revenue for 1 hour participation is higher than electricity costs for the entire day. Further, the revenue from DR participation is higher for the multi-step bidding approach, compared to both one step bidding and benchmark case, as a result of the higher LCQ.

\section{CONCLUSION}

An novel TE-based control where multiple air-conditioned zones in a building transact for conditioned air in a virtual market is proposed for air conditioning systems in buildings to enable their participation in DR markets. The proposed multi-step bidding approach for VAV agents is integrated with whole-building energy modeling platform (i.e. EnergyPlus) and evaluated through simulation studies performed in MATLAB-EnergyPlus co-simulation environment. Moreover, the comparison of non-DR activation and DR activation cases is presented to establish the benefits of the proposal. It is observed that in case of DR activations, each floor may be able to contribute significant capacity (up to $56 \%$ ) in demand reduction for 1 hour, while simultaneously respecting occupant comfort preferences. The formation of demand-side bid to participate in Singapore EMA's Demand Response program is also presented along with revenue estimation for a case study considering real electricity market prices.

The scope for future work includes extension for other ancillary service markets or BEM platforms.

\section{REFERENCES}

[1] U.S. Energy Information Administration, "Electric Power Annual 2019," Feb. 2019. [Online]. Available: https://www.eia.gov/electricity/ annual/pdf/epa.pdf

[2] Energy Market Authority Singapore, "EMA | Singapore Energy Statistics - Energy Consumption 2019." [Online]. Available: https: //www.ema.gov.sg/singapore-energy-statistics/Ch03/index3

[3] K. Chua, S. Chou, W. Yang, and J. Yan, "Achieving better energyefficient air conditioning a review of technologies and strategies," Applied Energy, vol. 104, pp. 87 - 104, 2013.

[4] N. Lu, "An Evaluation of the HVAC Load Potential for Providing Load Balancing Service," IEEE Transactions on Smart Grid, vol. 3, no. 3, pp. 1263-1270, 2012.

[5] A. Wierman, Z. Liu, I. Liu, and H. Mohsenian-Rad, "Opportunities and challenges for data center demand response," in International Green Computing Conference, Nov 2014, pp. 1-10.

[6] Energy Market Authority of Singapore, "Demand Response Programme," [Online]. Available: https://www.ema.gov.sg/Demand_ Response_Program.aspx., 2018, [Accessed: 28 Sep 2018].

[7] K. Oikonomou, M. Parvania, and R. Khatami, "Optimal Demand Response Scheduling for Water Distribution Systems," IEEE Transactions on Industrial Informatics, vol. 14, no. 11, pp. 5112-5122, 2018.
[8] The GridWise Architecture Council, "GridWise Transactive Energy Framework Version 1.1," July 2019.

[9] K. Kok and S. Widergren, "A Society of Devices: Integrating Intelligent Distributed Resources with Transactive Energy," IEEE Power and Energy Magazine, vol. 14, no. 3, pp. 34-45, May 2016.

[10] EMA Singapore, "Implementing Demand Response in the National Electricity Market of Singapore - Final Determination Paper,' [Online]. Available: https://www.ema.gov.sg/cmsmedia/Electricity/Demand Response/Final_Determination_Demand_Response_28_Oct_2013_ Final.pdf, [Accessed: June 8, 2018].

[11] J. H. Yoon, R. Baldick, and A. Novoselac, "Dynamic Demand Response Controller Based on Real-Time Retail Price for Residential Buildings," IEEE Transactions on Smart Grid, vol. 5, no. 1, pp. 121-129, Jan 2014.

[12] X. Zhou, T. Hong, and D. Yan, "Comparison of building energy modeling programs: Hvac systems." [Online]. Available: https: //www.osti.gov/biblio/1165199

[13] J. Reynolds, Y. Rezgui, A. Kwan, and S. Piriou, "A zone-level, building energy optimisation combining an artificial neural network, a genetic algorithm, and model predictive control," Energy, vol. 151, pp. 729 739, 2018. [Online]. Available: http://www.sciencedirect.com/science/ article/pii/S036054421830522X

[14] Y. J. Kim, "A Supervised-Learning-Based Strategy for Optimal Demand Response of an HVAC System in a Multi-Zone Office Building," IEEE Transactions on Smart Grid, vol. 11, no. 5, pp. 4212-4226, 2020.

[15] D. B. Crawley, C. O. Pedersen, L. K. Lawrie, and F. C. Winkelmann, "Energyplus: Energy simulation program," ASHRAE Journal, vol. 42, pp. 49-56, 2000.

[16] B. Liu, M. Akcakaya, and T. E. Mcdermott, "Automated control of transactive hvacs in energy distribution systems," IEEE Transactions on Smart Grid, vol. 12, no. 3, pp. 2462-2471, 2021.

[17] N. A. Daher, M. Lattouf, I. Mougharbel, M. Saad, H. Y. Kanaan, M. Raoofat, D. Asber, and J. Beltran-Galindo, "Improved control method of HVAC system for Demand Response," in 2018 4th International Conference on Renewable Energies for Developing Countries (REDEC), Nov 2018, pp. 1-6.

[18] M. Ostadijafari, A. Dubey, and N. Yu, "Linearized Price-Responsive HVAC Controller for Optimal Scheduling of Smart Building Loads," IEEE Transactions on Smart Grid, vol. 11, no. 4, pp. 3131-3145, 2020.

[19] H. Hao, C. D. Corbin, K. Kalsi, and R. G. Pratt, "Transactive Control of Commercial Buildings for Demand Response," IEEE Transactions on Power Systems, vol. 32, no. 1, pp. 774-783, Jan 2017.

[20] Office of Energy Efficiency \& Renewable Energy, "EnergyPlus Project Impact," 2014. [Online]. Available: https://www.energy.gov/ eere/buildings/downloads/energyplus-0

[21] R. Chandra, G. N. B. Yadav, and S. K. Panda, "Transactive Control of Air-Conditioning Systems in Buildings for Demand Response," in 2020 IEEE PES Innovative Smart Grid Technologies Europe (ISGT-Europe), 2020, pp. 81-85.

[22] S. Zhou, Z. Shu, Y. Gao, H. B. Gooi, S. Chen, and K. Tan, "Demand response program in singapore's wholesale electricity market," Electric Power Systems Research, vol. 142, pp. 279 - 289, 2017. [Online]. Available: http://www.sciencedirect.com/science/article/ pii/S0378779616303741

[23] Energy Market Company. Price Information. [Online]. Available: https://www.emcsg.com/MarketData/PriceInformation

[24] M. Deru et al., "U.S. Department of Energy commercial reference building models of the national building stock," Publications (E), no. February 2011, pp. $1-118,2011$. [Online]. Available: https://www.nrel.gov/docs/fy11osti/46861.pdf

[25] National Renewable Energy Laboratory, "Weather Data by Location | EnergyPlus," [Accessed: May 31 2021]. [Online]. Available: https://energyplus.net/weather

[26] U. S. Department of Energy, "Energyplus Engineering Reference," 2020. [Online]. Available: https://energyplus.net/sites/all/modules/ custom/nrel_custom/pdfs/pdfs_v9.4.0/EngineeringReference.pdf

[27] Duarte Antunes, "Optimal Control and Dynamic Programming: Lecture 2," [Online]. Available: http://www.dct.tue.nl/New/Antunes/Slides/ Lecture2.pdf, [Accessed: Nov. 29, 2020].

[28] J. A. Pinzon, P. P. Vergara, L. C. P. da Silva, and M. J. Rider, "Optimal management of energy consumption and comfort for smart buildings operating in a microgrid," IEEE Transactions on Smart Grid, vol. 10, no. 3, pp. 3236-3247, 2019.

[29] J. Dostal and T. Baumelt, "Model predictive control for buildings with active one-pipe hydronic heating," E3S Web of Conferences, vol. 111, p. 04050, 2019. [Online]. Available: https://doi.org/10.1051\%2Fe3sconf\% 2F201911104050 